\title{
Anderson-Yuval approach to the multichannel Kondo problem
}

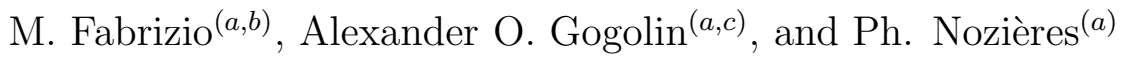 \\ (a) Institut Laue-Langevin, B.P.156 38042 Grenoble, Cedex 9, France \\ ${ }^{(b)}$ Institut for Advanced Studies, Via Beirut 4, 34014 Trieste, Italy \\ (c) Landau Institute for Theoretical Physics, Kosygina str. 2, Moscow, 117940 Russia
}

(October 28, 2018)

\begin{abstract}
We analyze the structure of the perturbation expansion of the general multichannel Kondo model with channel anisotropic exchange couplings and in the presence of an external magnetic field, generalizing to this case the AndersonYuval technique. For two channels, we are able to map the Kondo model onto a generalized resonant level model. Limiting cases in which the equivalent resonant level model is solvable are identified. The solution correctly captures the properties of the two channel Kondo model, and also allows an analytic description of the cross-over from the non Fermi liquid to the Fermi liquid behavior caused by the channel anisotropy.
\end{abstract}

\section{INTRODUCTION}

The single channel Kondo model has a long history as the simplest model believed to contain the relevant physics of magnetic impurities embedded in metals. A lot of efforts have been devoted to study this model and presently one can safely claim that it has been completely understood from the theoretical point of view. Apart from the original perturbative scaling approach, which already gave the correct qualitative description㽢, there is also an exact solution of this model obtained by Bethe-ansatz technique 3 . The physics 
underlying the single channel Kondo model is the formation of a non degenerate singlet state at low temperature. The impurity spin is screened by the conduction electrons, hence the magnetic susceptibility, obeying Curie-Weiss law at high temperatures, undergoes the Kondo cross-over saturating to a constant upon lowering the temperature.

In an attempt to describe realistic magnetic impurities (which have orbital structure), different generalizations of the simple Kondo model have been proposed 1 . Among these generalizations, the simplest one is probably the model describing an impurity spin $S$ coupled to $N$ channels of conduction electrons (commonly referred to as the multichannel Kondo model). Surprisingly, it has been realised that, for $N>2 S$, this model exhibits a behavior qualitatively different from the single channel onel, which is due to a non-trivial ground state with a residual degeneracy. This gives rise to divergent low temperature susceptibility $\chi_{i m p}$ and specific heat coefficient $\gamma=C_{V, i m p} / T$ (so-called non Fermi liquid behavior). It should be said that the experiments on dilute magnetic alloys (the systems, for which the Kondo model was originally proposed) do not give any clear evidence for such a behavior but rather suggest that the ground state is always a singlet. A possible explanation would be that the channel symmetry (which is necessary for the non Fermi liquid behavior to occur) is broken (since typically no exact symmetry guarantees channel equivalence). If the energy scale of this symmetry breaking term is small, a slow cross-over from the non Fermi liquid to the Fermi liquid behavior is expected. However, it might be difficult to experimentally distinguish it from the usual Kondo cross-over. Consequently, since early 80's, the interest to the issue was decaying, even though, in the meanwhile, some exact solutions for the case of equivalent channels became available.

In recent years, however, interest to the subject grew again, as other, more promising, realizations of the multichannel Kondo model have been proposed; for instance, two level systems in metals 9 , heavy fermion compounds ${ }^{\text {G }}$, high Tc superconductors. While for the two level systems there are convincing experimental evidences ${ }^{2}$ of the non Fermi liquid behavior predicted by the theory, the other proposed realizations are till now controversial.

The general multichannel Kondo hamiltonian is given by 


$$
H_{K}=\sum_{a=1}^{N} \sum_{\sigma} H_{0}\left(\psi_{a \sigma}, \psi_{a \sigma}^{\dagger}\right)+\sum_{a=1}^{N}\left\{J_{z a} S^{z} \sigma_{a}^{z}(\mathbf{0})+\frac{J_{\perp a}}{2}\left[S^{+} \sigma_{a}^{-}(\mathbf{0})+S^{-} \sigma_{a}^{+}(\mathbf{0})\right]\right\}
$$

where

$$
H_{0}\left(\psi, \psi^{\dagger}\right)=\sum_{\mathbf{k}} \epsilon_{\mathbf{k}} \psi_{\mathbf{k}}^{\dagger} \psi_{\mathbf{k}}
$$

is the kinetic energy of the conduction electrons $\psi_{a \sigma}, a=1, \ldots, N$ being the channel index, and $\sigma=\uparrow, \downarrow$ being the spin index (we assume a spin $1 / 2$ impurity). Notice however that in some realizations of this model, the channel index is the physical spin while the spin index labels an orbital quantum number (see also Section IV). The electron spin densities in Eq. (1) are defined by

$$
\begin{aligned}
\sigma_{a}^{z}(\mathbf{x}) & =\frac{1}{2}\left[\psi_{a \uparrow}^{\dagger}(\mathbf{x}) \psi_{a \uparrow}(\mathbf{x})-\psi_{a \downarrow}^{\dagger}(\mathbf{x}) \psi_{a \downarrow}(\mathbf{x})\right] \\
\sigma_{a}^{+}(\mathbf{x}) & =\psi_{a \uparrow}^{\dagger}(\mathbf{x}) \psi_{a \downarrow}(\mathbf{x}) .
\end{aligned}
$$

In the single channel case $(N=1)$ the exchange couplings $J$ flow to infinity under scaling, if positive. The spin anisotropy $J_{z} \neq J_{\perp}$ disappears at the fixed point. This is interpreted as the formation of a singlet at the impurity site. On the contrary for the $N>1$ channel symmetric case, the infinite coupling fixed point is unstable as well as the weak coupling one, hence a stable intermediate coupling fixed point.

Until recently, the information on the behavior of the model around this fixed point could only be extracted from the Bethe-ansatz solution 10 and conformal field theory 11 . In 1992, Emery and Kivelson 12 provided a simple solution for the symmetric two channel case at a particular value of the longitudinal exchange coupling. The solution was obtained by means of abelian bosonization technique generalizing a procedure previously applied to the single channel Kondo model by Schlottmann13. The original Schlottmann's approach was in turn inspired by Toulouse's mapping of the single channel Kondo model onto a resonant level model, which he achieved by the analysis of the partition function 14 , borrowing the perturbative treatment of Anderson and Yuval15. The two methods, bosonization and Anderson-Yuval method, are equivalent, in the sense that they give qualitatively similar results (it has been checked in the single channel case). Still, the latter is formally more 
rigorous (e.g. it does not fully relies on band linearization) and straightforward (it simply amounts to compare the perturbation expansions of two different models).

In this paper we generalize the Anderson-Yuval method to the multichannel Kondo model with channel anisotropic exchange couplings. For the two channel case, we are able to show that the perturbation expansion of the Kondo model is equivalent to that of a generalized resonant level model. In the channel isotropic case, the model is of the type found by Emery and Kivelson via bosonization technique. The novel feature is the channel anisotropy which gives rise to interesting cross-over phenomena. We demonstrate that even in this case a mapping of the Kondo model (for particular values of the longitudinal exchange couplings) onto a solvable resonant level model does exist. For $N>2$ we are unable to find any kind of resonant level model which would reproduce the perturbation expansion.

\section{GENERALIZATION OF THE ANDERSON YUVAL APPROACH}

In this Section, we analyze the structure of the perturbation expansion of the hamiltonian (回) in the transverse exchange couplings.

\section{A. Single channel model}

Consider first the single channel problem. We allow for an exchange anisotropy $\left(J_{x}=\right.$ $J_{y}=J_{\perp}$ is different from $J_{z}$ ). We want to calculate the impurity partition function in time space, using a perturbation expansion in powers of $J_{\perp}$. A term of order $2 n$ involves $2 n$ alternate impurity spin flips. Let $t_{i}$ be the times of up flips, $t_{i}^{\prime}$ that of down flips $(n$

of each). The philosophy is to calculate that particular term exactly, for a given history $\left\{t_{i}, t_{i}^{\prime}\right\}$, and to show that it is identical to the corresponding expansion for another problem (a resonant level), with appropriately chosen parameters. The two problems are mapped on each other term by term: they are equivalent. Note that we thus bypass summation of the perturbation series in $J_{\perp}$. For a specific value of $J_{z}$ (the "Toulouse limit") the equivalent problem happens to be trivially solvable: if we can scale through that value we have an 
explicit description of the crossover to low temperature - the " $(100-\epsilon) \% "$ exact solution of Anderson. The error stems from the fact that universal scaling is not just a change of $J_{z}$. That error is supposed not to change the qualitative behavior - and anyway it is implicit in the equivalent bosonization technique (less powerful since it relies on a Born approximation for phase shifts).

Assume first that $J_{z}=0$. Each vertex flips a conduction electron spin. A $t_{i}$ vertex creates a $\downarrow$ electron and an $\uparrow$ hole, a $t_{i}^{\prime}$ vertex does the reverse. In a typical diagram, the electron propagators go from any $t_{i}$ to any $t_{i}^{\prime}$ for $\downarrow$ spins, and from any $t_{i}^{\prime}$ to any $t_{i}$ for $\uparrow$ spins. Since $J_{z}=0$ these propagators are free electron local propagators

$$
G_{0}(t)=\frac{i \nu_{0}}{t-i \xi_{0}^{-1} \operatorname{sign} t}
$$

where $\nu_{o}$ is the density of states for one spin at the Fermi level, and $\xi_{0}$ is a high energy cut-off of the order of the conduction bandwidth. Let $D_{\sigma}\left(t_{i}, t_{i}^{\prime}\right)$ be the contribution of the $n$ lines with spin $\sigma$ that join $t_{i}$ and $t_{i}^{\prime}$ vertices. $D_{\sigma}$ has a pole whenever $t_{i}=t_{j}^{\prime}$ (one propagator is divergent). Moreover crossing symmetry implies a zero whenever $t_{i}=t_{j}$ (or $t_{i}^{\prime}=t_{j}^{\prime}$ ): exchanging the extremities of two propagators changes the sign. Hence the Cauchy determinant found by Anderson and Yuval (here and in what follows we omit time independent pre-factors containing the density of states $\nu_{0}$ and to the short-time cut-off, restoring them in the final expressions)

$$
D_{\sigma}=\frac{\prod_{i<j}\left(t_{i}-t_{j}\right) \prod_{i<j}\left(t_{i}^{\prime}-t_{j}^{\prime}\right)}{\prod_{i, j}\left(t_{i}-t_{j}^{\prime}\right)}
$$

Expression (4) is homogeneous with degree $(-n)$, as expected for $D_{\sigma}$. The proof is completed by looking at the asymptotic behavior. The corresponding contribution to the impurity propagator is $U_{0}=D_{\uparrow} D_{\downarrow}$.

We now restore $J_{z}$. The potential felt by a spin $\sigma$ electron changes at each impurity flip - hence an edge singularity that will modify the long time behavior of $U$, see Ref. 16. In Fig.1 we have drawn the time dependent potential felt e.g. by the up-spin conduction 
electrons at the impurity site (the down-spin one is the opposite) The phase shift is $\delta_{+}$(resp. $\delta_{-}$) when the electron and impurity have parallel (resp. antiparallel) spins. What matters is the discontinuity of phase shifts when a flip occurs, $\delta=\delta_{+}-\delta_{-}$. If we assume electron hole symmetry, then

$$
\delta_{+}=-\delta_{-}=\tan ^{-1}\left(\pi \nu_{0} J_{z} / 4\right)
$$

The effect of the flip is two-fold:

(i) The open lines that contribute to $D_{\sigma}$ can scatter any number of times on the impurity. That generates once again the Cauchy determinant (via a Muskhelishvili type of analysi 17 ). For each spin $D_{\sigma}$ is replaced by $D^{1-2 \delta / \pi}$, thus open line contribution acquires an extra factor $U_{L}=D^{-4 \delta / \pi}$.

(ii) In addition the underlying Fermi sea reacts to the flip via closed loops that exponentiate (for a given history the potential is structureless). The resulting contribution is $U_{C}=$ $D^{2 \delta^{2} / \pi^{2}}$.

(iii) Altogether $U=U_{0} U_{L} U_{C}$, and, after inserting back the prefactors, we obtain

$$
U=\left(\frac{J_{\perp} \nu_{0} \xi_{0}}{2}\right)^{2 n}\left(\frac{D^{\eta}}{\xi_{0}^{n}}\right)^{\eta}
$$

where $\eta$ is an exponent that depends on $J_{z}$,

$$
\eta=2\left(1-\frac{\delta}{\pi}\right)^{2} .
$$

(Note that $\eta=0$ if $\delta=\pi$, which corresponds to the strong coupling limit $\delta_{+}=-\delta_{-}=$ $\pi / 2)$.

Let us now consider a resonant level model for spinless electrons, characterized by the hamiltonian

$$
H=H_{0}\left(\Psi, \Psi^{\dagger}\right)+\lambda\left[\Psi^{\dagger}(\mathbf{0}) d+d^{\dagger} \Psi(\mathbf{0})\right]+\frac{V}{2}\left[\Psi^{\dagger}(\mathbf{0}) \Psi(\mathbf{0})-\Psi(\mathbf{0}) \Psi^{\dagger}(\mathbf{0})\right]\left(d^{\dagger} d-\frac{1}{2}\right)
$$


where $d$ is an impurity orbital at the Fermi energy located at the origin. $\Psi$ is a free Fermi field which kinetic energy is the same as in Eq.(11). The interaction potential $V$ produces a phase shift discontinuity

$$
\delta^{\prime}=2 \tan ^{-1}\left(\frac{\pi \nu_{0} V}{2}\right)
$$

between the empty and full d-states. We expand in powers of $\lambda$ which plays the role of $J_{\perp}$. The $t_{i}$ and $t_{i}^{\prime}$ vertices correspond to $d^{\dagger}$ and $d$ operators. The structure of the expansion is the same as for the Kondo case, except that there is no spin degeneracy. We have one set of open lines originating from $\lambda$ vertices, which can scatter off the flipping $\delta^{\prime}$. We also have one set of closed loops - hence altogether

$$
U^{\prime}=\left(\lambda \sqrt{\nu_{0} \xi_{0}}\right)^{2 n}\left(\frac{D}{\xi_{0}^{n}}\right)^{\eta^{\prime}}
$$

with

$$
\eta^{\prime}=\left(1-\frac{\delta^{\prime}}{\pi}\right)^{2}
$$

Thus the Kondo problem with coupling $\delta$ is mapped term by term onto the resonant level with coupling $\delta^{\prime}$ if the two propagators Eqs .(6) and (9) are identical. This implies $\eta=\eta^{\prime}$ (that can always be achieved by appropriately choosing $V$ ) and

$$
\lambda=\frac{J_{\perp}}{2} \sqrt{\nu_{0} \xi_{0}}
$$

The Toulouse limit corresponds to $\delta^{\prime}=0$, i.e. a phase shift $\delta=\pi(1-1 / \sqrt{2})$. The resonant level hamiltonian can then be trivially diagonalized, yielding a low temperature Fermi liquid behavior. The resonant level hamiltonian of the form (7) has been previously derived by Wiegmann and Finkelstien 18.

\section{B. Multichannel model}

We now turn to the $\mathrm{N}$ channel case. In a first stage we assume flavor degeneracy: what does remain of the previous analysis? In order to answer that question we proceed in reverse. 
(i) The alternation of up and down spins is unchanged. The flipping potential due to $\delta$ is the same as before (see Fig.1), whatever the flavor involved at each vertex. Edge singularities are consequently unaffected. The scattering contribution to open lines is again $U_{L}=D^{-4 \delta / \pi}$ (flavor is fixed by extremities). Each closed loop can have an arbitrary flavor and therefore $U_{C}=D^{2 N \delta^{2} / \pi^{2}}$.

(ii) Paradoxically the difficulties come from the part $U_{0}$ (in the absence of $J_{z}$ ). $U_{0}$ still has poles whenever $t_{i}=t_{j}^{\prime}$, but one looses crossing symmetry. If the ends of two lines are interchanged one usually changes the number of closed loops $\mathrm{C}$ - hence a change in the degeneracy $N^{C}$. As a result $U_{0}$ cannot be expressed simply in terms of $D$.

In order to proceed, we must assign to each vertex its flavour index $a$. The $t_{i}$ and $t_{j}^{\prime}$ then break into $N$ subclasses, $t_{i a}$ and $t_{j a}^{\prime}(a=1, \ldots, N)$. For a given diagram the number of vertices in different subclasses needs not be the same, but spin and flavor conservation implies an equal number of $t_{i a}$ and $t_{j a}^{\prime}$ within a given subclass. Since flavor is conserved along an open line, $U_{0}$ is a product of independent factors $U_{0 a}$. For each factor crossing symmetry holds and $U_{0 a}$ is the square of a Cauchy determinant $D_{a}$ as it would be for a single channel. $D_{a}$ is still given by (田), the products running only over $a$-type times, $t_{i a}$ and $t_{j a}^{\prime}$. In the end we find (again omitting prefactors proportional to $J_{\perp}, \nu_{0}$ and $\xi_{0}$ )

$$
U=\left[D_{1} \ldots D_{N}\right]^{2} D^{-4 \frac{\delta}{\pi}+2 N\left[\frac{\delta}{\pi}\right]^{2}}=\frac{\left[D_{1} \ldots D_{N}\right]^{2}}{D} D^{\beta_{N}}
$$

where

$$
\beta_{N}=1-4 \frac{\delta}{\pi}+2 N\left(\frac{\delta}{\pi}\right)^{2}
$$

Note that $D$ is not the product of individual $D_{a}$ : we have instead $D=D_{1} \ldots D_{N} F$, in which we have set

$$
F=\frac{\prod_{i j} \prod_{a<b}\left(t_{i a}-t_{j b}\right)\left(t_{i a}^{\prime}-t_{j b}^{\prime}\right)}{\prod_{i j} \prod_{a<b}\left(t_{i a}-t_{j b}^{\prime}\right)\left(t_{i a}-t_{j b}^{\prime}\right)}
$$

The factor $F$ couples the channels. 
The Emery-Kivelson solution to the two channel case, $N=2$, is based on a mapping of the Kondo problem onto the following spinless resonant level hamiltonian

$$
\begin{aligned}
H & =H_{0}\left(\Psi, \Psi^{\dagger}\right)+H_{0}\left(\Psi_{s}, \Psi_{s}^{\dagger}\right)+\lambda\left(d^{\dagger}-d\right)\left[\Psi^{\dagger}(\mathbf{0})+\Psi(\mathbf{0})\right] \\
& +\frac{V}{2}\left[\Psi_{s}^{\dagger}(\mathbf{0}) \Psi_{s}(\mathbf{0})-\Psi_{s}(\mathbf{0}) \Psi_{s}^{\dagger}(\mathbf{0})\right]\left(d^{\dagger} d-\frac{1}{2}\right)
\end{aligned}
$$

where $d$ is again a fictitious spinless Fermi operator. Notice that we have introduced two Fermi fields $\Psi$ and $\Psi_{s}$, coupled to the impurity in a different way (the reason why we have not used the same field will become clear later). In order to establish the equivalence we first consider the case $V=0$. We divide the $d$ and $d^{\dagger}$ flips into two subclasses, depending on whether a fermion $\Psi$ is emitted or absorbed. Times $t_{i 1}$ and $t_{i 2}$ correspond to $d^{\dagger}$ flips with a fermion emitted or absorbed respectively, $t_{i 1}^{\prime}$ and $t_{i 2}^{\prime}$ are their hermitian conjugates. A spinless fermion propagator can go as usual from $t_{i 1}$ to $t_{j 1}^{\prime}\left(t_{i 2}^{\prime}\right.$ to $\left.t_{j 2}\right)$ or from $t_{i 1}$ to $t_{j 2}\left(t_{i 2}^{\prime}\right.$ to $\left.t_{j 1}^{\prime}\right)$. The latter possibility is the new feature. The corresponding impurity propagator $U^{\prime}$ has poles whenever a propagator has zero time range, i.e. when $t_{i a}=t_{j a}^{\prime}, t_{i 1}=t_{j 2}$ or $t_{i 2}^{\prime}=t_{j 1}^{\prime}$. Due to crossing symmetry it has zeroes when $t_{i a}=t_{j a}, t_{i a}^{\prime}=t_{j a}^{\prime}, t_{i 1}=t_{j 2}^{\prime}$ or $t_{i 1}^{\prime}=t_{j 2}$. Once again one thereby builds a Cauchy determinant which happens to be

$$
U^{\prime}=\frac{D_{1} D_{2}}{F}
$$

Expression (13) has the right poles and zeroes. It moreover has the right overall power of $t$ and asymptotic behavior: it is the correct answer. Comparing (13) with the definition of $F$ we see that

$$
U^{\prime}=\frac{\left(D_{1} D_{2}\right)^{2}}{D}
$$

We now restore the flipping potential $V$. Since it involves a different Fermi field, it gives rise only to a closed loop contribution. Altogether we have

$$
U^{\prime}=\frac{\left(D_{1} D_{2}\right)^{2}}{D} D^{\left(\frac{\delta^{\prime}}{\pi}\right)^{2}}
$$

with the same $\delta^{\prime}$ as in Eq. (8). Comparing (14) with (10) we see that the two problems are mapped onto each other if [see Eq. (11)] 


$$
\beta_{2}=\left(1-2 \frac{\delta}{\pi}\right)^{2}=\left(\frac{\delta^{\prime}}{\pi}\right)^{2}
$$

which is always possible since both right and left sides are positive. Notice that for a given $\delta$ the interaction potential $V$ in Eq. (12) is, according to (8) and (15), given by

$$
V=\frac{2}{\pi \nu_{0}} \tan \left(\frac{\delta^{\prime}}{2}\right)=\frac{2}{\pi \nu_{0}} \tan \left(\frac{\pi}{2}-\delta\right)
$$

The problem is directly solvable if $V=0$, i.e. when $\delta=\pi / 2$. In the electron hole symmetric case that implies $\delta_{+}=-\delta_{-}=\pi / 4$ - a typical intermediate coupling as expected for the two channel overscreened Kondo impurity. Indeed from the expression of $\beta_{2}$ we see that the model is symmetric under $\delta \rightarrow \pi-\delta$. This extends the result of Ref. 5 that the two channel Kondo model behaves similarly around $J_{z}=0$ (i.e. $\delta=0$ ) and $J_{z}=\infty$ (i.e. $\delta=\pi)$. By symmetry the fixed point should be exactly at $\delta=\pi / 2$, that is at the solvable line $V=0$.

The argument can be extended to a flavor dependent exchange $J$. Due to anisotropy we must treat separately the channel dependence of $J_{\perp}$ and $J_{z}$. Different $J_{\perp 1}$ and $J_{\perp 2}$ do not affect the structure of the perturbation expansion. As we have shown above the mapping works as follows:

$$
\begin{aligned}
& \Psi_{1 \downarrow}^{\dagger}\left(\mathbf{0}, t_{i 1}\right) \Psi_{1 \uparrow}\left(\mathbf{0}, t_{i 1}\right) S^{+}\left(t_{i 1}\right) \longmapsto d^{\dagger}\left(t_{i 1}\right) \Psi\left(\mathbf{0}, t_{i 1}\right) ; \\
& \Psi_{2 \downarrow}^{\dagger}\left(\mathbf{0}, t_{i 2}\right) \Psi_{2 \uparrow}\left(\mathbf{0}, t_{i 2}\right) S^{+}\left(t_{i 2}\right) \longmapsto d^{\dagger}\left(t_{i 2}\right) \Psi^{\dagger}\left(\mathbf{0}, t_{i 2}\right) \text {; } \\
& \Psi_{1 \uparrow}^{\dagger}\left(\mathbf{0}, t_{i 1}^{\prime}\right) \Psi_{1 \downarrow}\left(\mathbf{0}, t_{i 1}^{\prime}\right) S^{-}\left(t_{i 1}^{\prime}\right) \longmapsto \Psi^{\dagger}\left(\mathbf{0}, t_{i 1}^{\prime}\right) d\left(t_{i 1}^{\prime}\right) ; \\
& \Psi_{2 \uparrow}^{\dagger}\left(\mathbf{0}, t_{i 2}^{\prime}\right) \Psi_{2 \downarrow}\left(\mathbf{0}, t_{i 2}^{\prime}\right) S^{-}\left(t_{i 2}^{\prime}\right) \longmapsto \quad \Psi\left(\mathbf{0}, t_{i 2}^{\prime}\right) d\left(t_{i 2}^{\prime}\right),
\end{aligned}
$$

thus we need only modify accordingly the flipping matrix elements of the equivalent model, which becomes

$$
\frac{J_{\perp 1}}{2} \sqrt{\nu_{0} \xi_{0}}\left[d^{\dagger} \Psi(\mathbf{0})+\Psi^{\dagger}(\mathbf{0}) d\right]+\frac{J_{\perp 2}}{2} \sqrt{\nu_{0} \xi_{0}}\left[d^{\dagger} \Psi^{\dagger}(\mathbf{0})+\Psi(\mathbf{0}) d\right]
$$

Notice that, if $J_{\perp 1}=J_{\perp 2}$, (17) reduces to (7) with $\lambda=J_{\perp 1} \sqrt{\nu_{0} \xi_{0}}$.

A difference between $J_{z 1}$ and $J_{z 2}$ gives rise to different phase shifts $\delta_{1}$ and $\delta_{2}$. Let us first consider the scattering correction to the "1" open line, $U_{L 1}$. The Muskhelishvili propagator for a channel 1 spin up electron is 


$$
G_{1}\left(t, t^{\prime}\right)=\frac{i \pi \nu_{0}}{t-t^{\prime}} \prod_{i, a}\left[\frac{\left(t-t_{i a}\right)\left(t^{\prime}-t_{i a}^{\prime}\right)}{\left(t-t_{i a}^{\prime}\right)\left(t^{\prime}-t_{i a}\right)}\right]^{-\frac{\delta_{1}}{\pi}}
$$

Its contribution to $U_{L 1}$ is obtained by putting $t$ equal to any $t_{i 1}, t^{\prime}$ to any $t_{i 1}^{\prime}$, hence a factor $\left[D_{1}^{2} F\right]^{-\delta_{1} / \pi}$. We square it in order to account for spin and we multiply by the corresponding term for channel 2. The closed line contribution is straightforward since flavor is conserved along a loop,

$$
U_{C}=D^{2\left(\delta_{1}^{2}+\delta_{2}^{2}\right) / \pi^{2}}
$$

Altogether the impurity propagator is

$$
U=D_{1}^{2-4 \delta_{1} / \pi} D_{2}^{2-4 \delta_{2} / \pi} F^{-2\left(\delta_{1}+\delta_{2}\right) / \pi} D^{2\left(\delta_{1}^{2}+\delta_{2}^{2}\right) / \pi^{2}}
$$

(Remember that $F=D / D_{1} D_{2}$.) If $\delta_{1}=\delta_{2}=\delta$, we recover the previous result (10).

In general let us write

$$
\delta_{1}=\delta+\varepsilon, \quad \delta_{2}=\delta-\varepsilon
$$

Then (18) reduces to

$$
U=\frac{\left(D_{1} D_{2}\right)^{2}}{D} D^{\beta_{2}} D^{4\left(\frac{\varepsilon}{\pi}\right)^{2}}\left(\frac{D_{2}}{D_{1}}\right)^{4 \frac{\varepsilon}{\pi}}
$$

The additional factors with respect to (14) can be reproduced with an extra potential

$$
\frac{W}{2}\left[\Psi^{\dagger}(\mathbf{0}) \Psi(\mathbf{0})-\Psi(\mathbf{0}) \Psi^{\dagger}(\mathbf{0})\right]\left(d^{\dagger} d-\frac{1}{2}\right)
$$

With the choice

$$
W=\frac{2}{\pi \nu_{0}} \tan \varepsilon=\frac{2}{\pi \nu_{0}} \tan \left(\frac{\delta_{1}-\delta_{2}}{2}\right)
$$

the closed loop contribution generates the factor $D^{(2 \varepsilon / \pi)^{2}}$. The last factor of (19) comes from the fact that the phase shift discontinuity is $+\varepsilon$ on the " 1 " vertices, $-\varepsilon$ on the "2" vertices (see Fig. 2). In that way one can map any version of the two channel Kondo impurity onto an extended Emery-Kivelson hamiltonian. 
It is interesting to examine to what extent such an analysis could be pursued if $N>2$. We return to the flavor symmetric case, for which (7) holds. If we manage to have $\beta_{N}=0$, then

$$
U=\frac{\left(D_{1} \ldots D_{N}\right)^{2}}{D}=\frac{D_{1} \ldots D_{N}}{F}
$$

Hence two questions: ( $i$ ) Can we achieve $\beta=0$ ? ( $i i$ ) If we can, is there a solvable model that gives the same $U$ ? It is clear that no real phase shift $\delta$ will achieve $\beta=0$ if $N>2$. That may be a definitive objection since poor man's scaling scans the real $\delta$ axis. Let us ignore it, hoping that some analytic continuation argument might help. Then in order to reproduce (21) we must introduce a coupling

$$
S^{+}\left(\Psi_{1}+\ldots+\Psi_{N}\right)+\text { H.c. }
$$

in which the $\Psi_{a}$ operators are such that the corresponding propagators are

$$
\begin{aligned}
& \left\langle\Psi_{a}(t) \Psi_{b}^{\dagger}\left(t^{\prime}\right)\right\rangle=g\left(t-t^{\prime}\right)\left(1-\delta_{a b}\right) \\
& \left\langle\Psi_{a}(t) \Psi_{b}\left(t^{\prime}\right)\right\rangle=g\left(t-t^{\prime}\right) \delta_{a b}
\end{aligned}
$$

$\left(g(t) \approx 1 / t\right.$ is the free electron propagator). Then $U$ will have poles whenever $t_{i a}=t_{j a}^{\prime}$ on

the one hand, $t_{i a}=t_{j b}, t_{i a}^{\prime}=t_{j b}^{\prime}(a \neq b)$ on the other. It will have zeroes if $t_{i a}=t_{j a}, t_{i a}^{\prime}=t_{j a}^{\prime}$ or $t_{i a}=t_{j b}^{\prime}$. That just generates the combination (21). It remains to be seen what kind of algebra could produce (22): we do not know of any.

\section{MAGNETIC FIELD EFFECTS}

Let us consider the effects of an uniform magnetic field $\vec{B}=(0,0, B)$ in the framework of the Anderson-Yuval approach. The magnetic field appears in the hamiltonian with a term

$$
H_{B}=-\mu_{B} B\left[g_{i} S^{z}+g_{c} \int d \mathbf{x} \sum_{a=1}^{N} \sigma_{a z}(\mathbf{x})\right]
$$

where the electron spin density is defined in Eq. (3), $g_{i}$ and $g_{c}$ are the Landé factors of the impurity and the conduction electrons respectively, and $\mu_{B}$ is the Bohr magneton. 
As before we will treat the transverse exchange perturbatively. This implies that the reference states $|\uparrow\rangle$ and $|\downarrow\rangle$, which are used for the perturbation expansion, are the eigenstates of the hamiltonian with fixed impurity spin direction in the presence of the magnetic field

$$
H_{\uparrow / \downarrow}=\sum_{a \sigma} H_{0}\left(\psi_{a \sigma}^{\dagger}, \psi_{a \sigma}\right) \pm \sum_{a=1}^{N} \frac{J_{a z}}{2} \sigma_{a z}(\mathbf{0}) \mp \frac{g_{i} \mu_{B}}{2} B-g_{c} \mu_{B} B \sum_{a=1}^{N} \int d \mathbf{x} \sigma_{a z}(\mathbf{x}) .
$$

We have to understand how the magnetic field modifies the perturbation expansion in $J_{\perp} . B$ gives rise to two effects.

(i) It shifts the chemical potential for up and down spin electrons (in opposite directions). This causes a small change in the spin up and down phase shifts if the band has a finite curvature at the Fermi energy. This effect is negligible at low temperature.

(ii) It causes a difference $\Delta E=E_{\uparrow}-E_{\downarrow}$ in the ground state energies of (24) for the two impurity spin directions, which appears in the Muskhelishvili propagators.

By standard phase shift arguments, based on Friedel's sum rule for the displaced charge, we find

$$
\Delta E=-\mu_{B} g_{i} B+\frac{1}{\pi} \sum_{a=1}^{N} \int_{\epsilon_{F}-\frac{g_{c} \mu_{B}}{2} B}^{\epsilon_{F}+\frac{g_{c} \mu_{B}}{2} B} d \epsilon \delta_{a}(\epsilon)
$$

where $\epsilon_{F}$ is the Fermi energy. For small magnetic field (25) reduces to

$$
\Delta E=-\mu_{B} g_{i} B+\frac{g_{c} \mu_{B}}{\pi} B \sum_{a=1}^{N} \delta_{a}
$$

The above energy difference enters in the impurity propagator (10) via the following phase factor

$$
\exp \left[-i \Delta E \sum_{i=1}^{n}\left(t_{i}-t_{i}^{\prime}\right)\right]
$$

The conduction electron part of $\Delta E$ actually represents the leading term of closed loops diagrams, that one which grows linearly with $\left(t_{i}-t_{i}^{\prime}\right)$ instead of logarithmically.

Which term has to be added to the resonant level model in order to reproduce (27)? It is easy to realize that the corresponding term is simply 


$$
\Delta E S_{z} \longmapsto \Delta E\left(d^{\dagger} d-\frac{1}{2}\right)
$$

Notice that at the Emery-Kivelson line for the two channel case

$$
\sum_{a=1,2} \delta_{a}=\pi
$$

so that if $g_{i}=g_{c}$ then $\Delta E=0$ (at first order in $B$ ). Consequently the impurity magnetic susceptibility vanishes, in agreement with conformal field theory 1 and bosonization approaches 19 . This in turns means that at the Emery-Kivelson line the reference states are such as to perfectly screen the impurity spin. When the departure away from the EmeryKivelson line is treated as a perturbation 19 , both the specific heat and susceptibility acquire logarithmic sigularities, leading to the universal Wilson ration $R_{W}=8 / 3$.

As to the $N>2$ channel symmetric case, conformal field theory 11 and abelian bosonization approaches (which till now exist only for $N=4$, see Ref. 20) again predict the impurity susceptibility to vanish at the fixed point. From Eq. (26), we see that $\Delta E=0$ for $\delta=\pi / N$, and therefore the impurity susceptibility is rigorously zero. If this is the true property of the fixed point, as it follows from the analysis of Ref. 11, then $\delta=\pi / N$ is the fixed point.

\section{SOLUTION OF THE TWO CHANNEL ANISOTROPIC MODEL}

In this section we discuss the two channel Kondo model in more detail, focusing on the effects of channel anisotropy. The starting hamiltonian is

$$
H_{K}=\sum_{a=1}^{2} \sum_{\sigma} H_{0}\left(\psi_{a \sigma}, \psi_{a \sigma}^{\dagger}\right)+\sum_{a=1}^{2}\left\{J_{z a} S^{z} \sigma_{a}^{z}(\mathbf{0})+\frac{J_{\perp a}}{2}\left[S^{+} \sigma_{a}^{-}(\mathbf{0})+S^{-} \sigma_{a}^{+}(\mathbf{0})\right]\right\} .
$$

If the exchange couplings are channel symmetric $J_{z 1}=J_{z 2}$ and $J_{\perp 1}=J_{\perp 2}$, it is known 5 that the hamiltonian (29) flows towards a non trivial fixed point. At this fixed point the model exhibits non Fermi liquid behavior, namely the impurity susceptibility $\chi_{i m p}$ and the specific heat over temperature $C_{V, i m p} / T$ diverge at low temperatures as $\ln (1 / T)$, and the zero

temperature entropy is finite and equal to $\ln (2) / 2$, as if half of the impurity spin degrees of freedom were decoupled from the conduction electrons. Physically this occurs because two 
(and more) channels tend to overscreen the impurity spin, so that the complete screening characteristic of the single channel Kondo model can not take place, thus leaving a ground state degeneracy. In the case of a finite channel anisotropy, the system will always choose the channel with the strongest exchange to screen the impurity spin, and the usual Fermi liquid behavior of the single channel model will finally take place at zero temperature. The corresponding RG flow diagram is sketched in Fig.3.

As we have shown in the previous sections, this hamiltonian (29) can be mapped onto the following resonant level hamiltonian

$$
\begin{aligned}
H_{R L} & =H_{0}\left(\Psi, \Psi^{\dagger}\right)+H_{0}\left(\Psi_{s}, \Psi_{s}^{\dagger}\right)+\frac{J_{\perp 1}}{2} \sqrt{\nu_{0} \xi_{0}}\left[d^{\dagger} \Psi(\mathbf{0})+\Psi^{\dagger}(\mathbf{0}) d\right] \\
& +\frac{J_{\perp 2}}{2} \sqrt{\nu_{0} \xi_{0}}\left[d^{\dagger} \Psi^{\dagger}(\mathbf{0})+\Psi(\mathbf{0}) d\right]+\frac{W}{2}\left[\Psi^{\dagger}(\mathbf{0}) \Psi(\mathbf{0})-\Psi(\mathbf{0}) \Psi^{\dagger}(\mathbf{0})\right]\left(d^{\dagger} d-\frac{1}{2}\right) \\
& +\frac{V}{2}\left[\Psi_{s}^{\dagger}(\mathbf{0}) \Psi_{s}(\mathbf{0})-\Psi_{s}(\mathbf{0}) \Psi_{s}^{\dagger}(\mathbf{0})\right]\left(d^{\dagger} d-\frac{1}{2}\right)+\Delta E\left(d^{\dagger} d-\frac{1}{2}\right)
\end{aligned}
$$

where the interaction potentials are related to the longitudinal exchange couplings via [see Eqs.(5)-(16)-(20)]

$$
\begin{aligned}
& W=\frac{1}{2} \frac{J_{z 1}-J_{z 2}}{1+\pi^{2} \nu_{0}^{2} J_{z 1} J_{z 2} / 16}, \\
& V=\frac{8}{\pi^{2} \nu_{0}^{2}} \frac{1-\pi^{2} \nu_{0}^{2} J_{z 1} J_{z 2} / 16}{J_{z 1}+J_{z 2}}
\end{aligned}
$$

and (assuming equal impurity and conduction electron Landé factors $g_{i}=g_{c}=g$ )

$$
\Delta E=-\frac{2 g \mu_{B}}{\pi} \tan ^{-1}\left(\frac{\pi \nu_{0} V}{2}\right) B \equiv-g \mu_{B} \Lambda(V) B
$$

In the case of symmetric exchange couplings, the resonant level model (30) reduces to the hamiltonian (12) originally considered by Emery and Kivelson. Then the combination $d^{\dagger}+d$ is decoupled from the conduction electrons (hence the ground state degeneracy and the non Fermi liquid behavior). A finite channel anisotropic transverse exchange couples this combination to conduction electrons and moves the system away from the non Fermi liquid fixed point towards the Fermi liquid single channel fixed point 21 . The smaller is the anisotropy, the lower is the cross-over temperature. In what follows we analytically study this cross-over in the solvable limit $V=W=0$. 
Since the total number of fermions is not conserved by the hamiltonian, there are anomalous Green functions. In the Nambu representation

$$
D=\left(\begin{array}{c}
d \\
d^{\dagger}
\end{array}\right)
$$

the impurity Green function

$$
\hat{G}_{d}(t)=-i\left\langle T\left(D(t) D^{\dagger}(0)\right)\right\rangle
$$

is a $2 \times 2$ matrix. Its Fourier transform can easily be evaluated. For $\omega$ much smaller than the bandwidth, we find:

$$
\hat{G}_{d}(\omega)=\frac{1}{2} \frac{\hat{\tau}_{0}-\hat{\tau}_{x}}{\omega+i \Gamma \operatorname{sign} \omega}+\frac{1}{2} \frac{\hat{\tau}_{0}+\hat{\tau}_{x}}{\omega+i \gamma \operatorname{sign} \omega},
$$

where the resonance widths are defined by

$$
\begin{aligned}
\Gamma & =\frac{\pi}{4} \nu_{0}^{2} \xi_{0}\left(J_{\perp 1}+J_{\perp 2}\right)^{2}, \\
\gamma & =\frac{\pi}{4} \nu_{0}^{2} \xi_{0}\left(J_{\perp 1}-J_{\perp 2}\right)^{2},
\end{aligned}
$$

$\hat{\tau}_{i}$ being the Pauli matrices, and $\hat{\tau}_{0}$ the unit matrix. The impurity spectral function is

$$
\hat{A}(\omega)=\frac{1}{2}\left(\hat{\tau}_{0}-\hat{\tau}_{x}\right) \frac{\Gamma}{\omega^{2}+\Gamma^{2}}+\frac{1}{2}\left(\hat{\tau}_{0}+\hat{\tau}_{x}\right) \frac{\gamma}{\omega^{2}+\gamma^{2}},
$$

and it is therefore equally shared by two lorentzians with different widths $\Gamma$ and $\gamma$. In the channel isotropic case $\gamma \rightarrow 0$, one of the two lorentzians tends to $\delta(\omega)$, representing the impurity degree of freedom which is decoupled from the conduction band in this particular $\operatorname{limit} 12$.

The impurity contribution to the free energy can be calculated in a standard way by integration over the coupling constant. The result is

$$
F(T)=F_{0}(T)+\int \frac{d \omega}{2 \pi} f(\omega)\left[\tan ^{-1}\left(\frac{\Gamma}{\omega}\right)+\tan ^{-1}\left(\frac{\gamma}{\omega}\right)\right]
$$

where $F_{0}(T)$ is the free energy in absence of coupling between the impurity and conduction electrons, $f(\omega)$ is the Fermi distribution function and the integral should be limited to the 
conduction bandwidth. The entropy can be calculated by $S(T)=-\partial F(T) / \partial T$. By defining the function

$$
\bar{S}(z)=\frac{1}{2 \pi z}\left[\psi\left(\frac{1}{2}+\frac{1}{2 \pi z}\right)-1\right]-\ln \Gamma\left(\frac{1}{2}+\frac{1}{2 \pi z}\right)+\frac{1}{2} \ln \pi
$$

where $\psi(z)$ is the psi-function and $\Gamma(z)$ is the gamma-function, the entropy turns out to be

$$
S(T)=\ln (2)+\bar{S}\left(\frac{T}{\Gamma}\right)+\bar{S}\left(\frac{T}{\gamma}\right)=\left\{\begin{array}{lr}
\frac{\pi T}{6}\left(\frac{1}{\Gamma}+\frac{1}{\gamma}\right), & T \ll \gamma \\
\ln \sqrt{2}, & \gamma \ll T \ll \Gamma \\
\ln 2-\frac{\Gamma+\gamma}{2 \pi T}, & T \gg \Gamma
\end{array}\right.
$$

the last equality being valid for $\gamma \ll \Gamma$. $S(T)$ is shown in Fig.4. We see that $S(0)=0$, as expected since no degeneracy is left, but there is a region of temperatures (the wider the smaller $\gamma$ is) where the entropy is close to that of the symmetric two channel model.

Another quantity of physical interest is the longitudinal impurity susceptibility. As we know from the above analysis, exactly on the Emery-Kivelson line, $\chi_{i m p}^{z z}=0$ and one has to consider deviations from this line (i.e. $V \neq 0$ ) in order to account for a finite impurity susceptibility 19 . The resulting susceptibility is

$$
\begin{aligned}
& \chi_{i m p}^{z z}=\left[g \mu_{B} \Lambda(V)\right]^{2} \int_{0}^{\beta} d \tau\left\langle T\left(S^{z}(\tau) S^{z}(0)\right)\right\rangle= \\
& {\left[g \mu_{B} \Lambda(V)\right]^{2} \frac{1}{\pi(\Gamma-\gamma)}\left[\psi\left(\frac{1}{2}+\frac{\Gamma}{2 \pi T}\right)-\psi\left(\frac{1}{2}+\frac{\gamma}{2 \pi T}\right)\right] .}
\end{aligned}
$$

In the case $\gamma \ll \Gamma$ the susceptibility shows the same kind of cross-over behavior as the entropy:

$$
\chi_{i m p}^{z z}=\left[g \mu_{B} \Lambda(V)\right]^{2} \cdot \begin{cases}\frac{1}{\pi(\Gamma-\gamma)} \ln \left(\frac{\Gamma}{\gamma}\right), & T \ll \gamma \\ \frac{1}{\pi(\Gamma-\gamma)} \ln \left(\frac{\Gamma}{T}\right), & \gamma \ll T \ll \Gamma \\ \frac{1}{4 T}, & T \gg \Gamma\end{cases}
$$

As expected the magnetic susceptibility saturates at low temperature, although at intermediate temperatures it shows the logarithmic behavior of the two-channel Kondo model.

It follows from (36) and (37) that the Wilson ration $R_{W}$ is not universal: it depends on the amount of anisotropy, $\gamma / \Gamma$. Such a conclusion is obvious in the limit of small anisotropy, 
when the energy scales are well separated. Then the residual entropy $\ln \sqrt{2}$ must be quenched in a temperature range $\sim \gamma$, implying $C_{V, i m p} \sim T / \gamma$, while the susceptibility $\chi_{\text {imp }}$ just rounds off logarithmic singularity, $\chi_{i m p} \sim \ln (\Gamma / \gamma)$ : the Wilson ratio is very small. Such a lack of universality is also apparent in the phenomenological, Fermi liquid description of the low temperature limit, $T \ll \gamma$. Then the impurity is quenched into a singlet, and the residual conduction electron phase shift in the channel $(m, \sigma)$ may be expanded as

$$
\delta_{m \sigma}(\epsilon)=\delta_{m 0}+\alpha_{m} \epsilon+\psi_{m} \delta n_{m,-\sigma}+\sum_{m^{\prime} \neq m} \phi_{m \sigma}^{m^{\prime} \sigma^{\prime}} \delta n_{m^{\prime} \sigma^{\prime}}
$$

where $\delta n_{m^{\prime} \sigma^{\prime}}$ is the change in the occupation measured from the ground state. Universality implies that $\delta_{m \sigma}(\epsilon)$ is invariant

(i) If the chemical potential of the other channel is changed (there is no channel flip)

(ii) If $\epsilon$ and the chemical potential are changed by the same amount (the Kondo singularity is attached to the Fermi level)

Hence in our two channel case

$$
\left\{\begin{array}{l}
\delta_{1 \sigma}=\delta_{10}+\alpha_{1}\left[\epsilon-\frac{\delta n_{1-\sigma}}{\nu_{s}}\right]+\theta_{1} \sigma \sigma^{\prime} \delta n_{2 \sigma^{\prime}} \\
\delta_{2 \sigma}=\delta_{20}+\alpha_{2}\left[\epsilon-\frac{\delta n_{2-\sigma}}{\nu_{s}}\right]+\theta_{2} \sigma \sigma^{\prime} \delta n_{1 \sigma^{\prime}}
\end{array}\right.
$$

(the cross terms $\theta_{1}, \theta_{2}$ are equal in the electron-hole symmetric case $\delta_{10}=\delta_{20}=\pi / 2$.) $\nu_{s}$ is the one channel density of $s$-states at the Fermi level. It is then straightforward to extend the analysis of Ref. 5: the resulting impurity corrections are

$$
\frac{C_{V, i m p}}{C_{V}}=\frac{\alpha_{1}+\alpha_{2}}{\pi \nu_{s}}, \quad \frac{\chi_{i m p}}{\chi}=\frac{2\left(\alpha_{1}+\alpha_{2}\right)}{\pi \nu_{s}}+\frac{\theta_{1}+\theta_{2}}{2 \pi}
$$

Due to the channel interaction $\theta$ the Wilson ratio $R_{W}=C_{V} \chi_{i m p} / \chi C_{V, i m p}$ departs from the single channel value 2. Put another way, one has a line of fixed points rather than a unique one. If " 1 " is the dominant screening channel, $J_{1}$ goes to infinity while $J_{2}$ may evolve towards any arbitrary value: once the spin $S$ is screened, $J_{2}$ no longer scales. This arbitrariness is reflected in the Wilson ratio. 
To our knowledge, the two channel Kondo model is most convincingly realized by two level systems in metal alloys 6 . This has recently been experimentally confirmed thanks to the development of point contact spectroscopye. In these systems, the role of the spin is played by some orbital degree of freedom, while the physical spin plays the role of the channel index. Thus the model is by construction channel isotropic. However, an external magnetic field breaks the channel symmetry and generates an effective channel anisotropy proportional to the curvature of the conduction electron band times the magnetic field $B$. In this case, the coupling to the magnetic field is described by the following term in the hamiltonian:

$$
H_{B}=-\frac{g_{c} \mu_{B}}{2} B \int d \mathbf{x} \sum_{\sigma}\left[\psi_{1 \sigma}^{\dagger}(\mathbf{x}) \psi_{1 \sigma}(\mathbf{x})-\Psi_{2 \sigma}^{\dagger}(\mathbf{x}) \Psi_{2 \sigma}(\mathbf{x})\right]
$$

where $\sigma$ is now the pseudo-spin index, and the channel indices 1 and 2 correspond to the physical spin up and down respectively. The magnetic field shifts the Fermi level of channel 1 with respect to that of channel 2. Electron-hole symmetry is thereby broken within each channel. It follows that the magnetic field induces a phase shift anisotropy, $\delta_{1}-\delta_{2} \propto B$, reflected into a finite $W \propto B$. Such a correction comes both from the correction to the Fermi level density of states

$$
\nu_{1}-\nu_{2}=g_{c} \mu_{B} \nu_{0}^{\prime} B, \quad \nu_{0}^{\prime}=\left.\frac{\partial \nu}{\partial \epsilon}\right|_{\epsilon=\epsilon_{F}}
$$

and from the Zeeman shift of band edges. The change of the density of states also modifies the pseudospin flip amplitudes in the equivalent resonant level model:

$$
\frac{J_{\perp}}{2} \sqrt{\nu_{1} \xi_{0}}\left[d^{\dagger} \Psi(\mathbf{0})+\Psi^{\dagger}(\mathbf{0}) d\right]+\frac{J_{\perp}}{2} \sqrt{\nu_{2} \xi_{0}}\left[d^{\dagger} \Psi^{\dagger}(\mathbf{0})+\Psi(\mathbf{0}) d\right]
$$

The hybridization anisotropy is equivalent to a finite $\gamma$

$$
\gamma=\frac{\pi}{16}\left(g \mu_{B}\right)^{2} \xi_{0} \frac{\left(\nu_{0}^{\prime}\right)^{2}}{\nu_{0}} J_{\perp}^{2} B^{2}
$$

Thus, $B$ causes the cross-over to a Fermi liquid behavior at low temperature as observed in Ref. 9. As to the physical magnetic susceptibility, it is related to the first derivative of the 
free energy (34) with respect to $\gamma$. The low temperature (low magnetic field) behavior of the susceptibility is given by:

$$
\chi_{i m p}=\frac{\left(g \mu_{B} \nu_{0}^{\prime}\right)^{2}}{16 \nu_{0}} \xi_{0} J_{\perp}^{2} \ln \left[\min \left(\frac{1}{T}, \frac{1}{B^{2}}\right)\right] .
$$

(One can show, that finite $W \propto B$ does not contribute to the log-divergent part of the susceptibility.)

Very recently, the channel anisotropic (but spin isotropic) Kondo model has been solved using Bethe ansatz methods by N. Andrei and A. Jerez22. Their conclusions are qualitatively similar ours.

We are thankful to N. Andrei for helpful discussions. 


\section{REFERENCES}

${ }^{1}$ P.W. Anderson, G. Yuval and D.R. Hamann Phys. Rev. B 1, 4664 (1970).

${ }^{2}$ K.G. Wilson, Rev. Mod. Phys. 47773 (1975); P. Nozières, J. Low Temp. Phys. 17, 31 (1974).

${ }^{3}$ N. Andrei, Phys. Rev. Lett. 45, 379 (1980); P.B. Wiegmann, JETP Lett. 31, 364 (1980).

${ }^{4}$ For a general review see A.C. Hewson, The Kondo Problem to Heavy Fermions, Cambridge University Press (1993). The early discussion of the orbital degeneracy may already be found in the appendix of the original Anderson paper [P.W. Anderson, Phys. Rev. 124, $41(1961)]$.

${ }^{5}$ P. Nozières and A. Blandin, J. Phys. (Paris) 41, 193 (1980).

${ }^{6}$ K. Vladár, G. Zimányi, and A. Zawadowski, Phys. Rev. Lett. 56, 286 (1986); A. Muramatsu and F. Guinea, Phys. Rev. Lett. 57, 2337 (1986).

${ }^{7}$ D.L. Cox, Phys. Rev. Lett. 59, 1240 (1987); C.L. Seaman, M.B. Maple, B.W.Lee, S. Ghamaty, M.S. Torikachvili, J.-S. Kang, L.Z. Lui, J.W. Allen, and D.L. Cox, ibid. 67, 2882 (1991); B. Andraka and A.M. Tsvelik, ibid. 67, 2886 (1991);

${ }^{8}$ D.L. Cox, M. Jarrell, C. Jayaprakash, H.R. Krishna-murty, and J. Deisz, Phys. Rev. Lett. 62, 2188 (1989); T. Giamarchi, C.M. Varma, A.E.Ruckenstein, and Ph. Nozières, ibid. 70, 3967 (1993); V.J. Emery and S. Kivelson, ibid. 71, 3701 (1993).

${ }^{9}$ D.C. Ralph and R.A. Buhrman, Phys. Rev. Lett. 69, 2118 (1992); D.C. Ralph, A.W.W. Ludwig, Jan von Delft and R.A. Buhrman, ibid. 72, 1064 (1994).

${ }^{10}$ N. Andrei and C. Destri, Phys. Rev. Lett. 52, 364 (1984); A. M. Tsvelik and P. B. Wiegmann, Z. Phys. B 54, 201 (1984).

${ }^{11}$ A.M. Tsvelik, J. Phys. C 2, 2833 (1990); I. Affleck and A. W. W. Ludwig, Nucl. Phys. B, 360, 641 (1991). 
${ }^{12}$ V.J. Emery and S. Kivelson, Phys. Rev. B 47, 10812 (1992).

${ }^{13}$ P. Schlottmann, J. Phys. (Paris) 6, 1486 (1978).

${ }^{14}$ G. Toulouse, C. R. Acad. Sci. 268, 1200 (1969).

${ }^{15}$ G. Yuval and P.W. Anderson, Phys. Rev. B 1, 1522 (1970).

${ }^{16}$ P. Nozières and C. De Dominicis, Phys. Rev. 178, 1097 (1969).

${ }^{17}$ N.I. Muskhelishvili, Singular Integral Equations, Noordhoff: P. Noordhoff Ltd., Groningen, The Netherlands (1953).

${ }^{18}$ P.B. Wiegmann and A.M. Finkelstein, Sov. Phys. JETP 48, 102 (1978).

${ }^{19}$ D.G. Clarke, T. Giamarchi and B.I. Shraiman, Phys. Rev. B 48, 7070 (1993); A.M. Sengupta and A. Georges, ibid. 49, 10020 (1994).

${ }^{20}$ M. Fabrizio and A.O. Gogolin, to appear in Phys. Rev. B.

${ }^{21}$ A different kind of cross-over is that induced by a local magnetic field. For the two channel case, this has been studied by D. Sacramento and P. Schlottmann, Phys. Lett. A 142, 245 (1989).

${ }^{22}$ N. Andrei and A. Jerez, Rutgers University preprint RU-94-94 and babbage, Nr.9412054. 


\section{FIGURES}

FIG. 1. Time-dependent potential seen by a spin up conduction electron. The impurity spin flips from $\downarrow$ to $\uparrow$ at times $t_{i}$ and vice versa at times $t_{j}^{\prime}$.

FIG. 2. Time-dependent local potential felt by an electron in the effective resonant level model. While the same annihilation operator $\Psi$ is involved in the two types of flips, the potentials are opposite. The flips at times $t_{i 1}^{\prime}$ and $t_{j 2}$ are the hermitian conjugates.

FIG. 3. Qualitative $\left(J_{1}, J_{2}\right)$ RG flow diagram for the anisotropic two channel Kondo model.

FIG. 4. Entropy $S(T)$ for various values of the anisotropy $\lambda^{2}=\gamma / \Gamma$ : from the top $\lambda=0,0.1,0.5,1$. 